\title{
CIRURGIA BARIÁTRICA EM ADOLESCENTES
}

\author{
Pedro Paulo Caravatto, Tarissa Petry, Ricardo Cohen
}

Centro de Obesidade - Hospital Infantil Sabará

Centro de Obesidade e Diabetes - Hospital Alemão Oswaldo Cruz

\section{RESUMO}

A obesidade na infância e adolescência tornou-se um grave problema de saúde pública. Doenças típicas do adulto relacionadas à obesidade, tais como diabetes tipo 2, hipertensão e dislipidemia, são cada vez mais prevalentes na população jovem, diminuindo consideravelmente a expectativa de vida. Desta forma, é preciso abordar o problema mais precocemente, a fim de evitar as graves consequencias dessas doenças a longo prazo. Neste contexto, a cirurgia bariátrica tem sido tema frequente no manejo da obesidade em indivíduos mais jovens.

O tratamento cirúrgico da obesidade em adolescentes apresenta riscos e benefícios a serem considerados e deve-se levar em conta determinadas características psicológicas e emocionais típicas do adolescente.

Os resultados iniciais da cirurgia bariátrica em adolescentes são promissores, semelhantes aos observados em adultos, entretanto há necessidade de maior tempo de seguimento para avaliá-los a longo prazo.

Neste capítulo, os autores discutem a epidemiologia e o impacto da obesidade infantil, as indicações do tratamento cirúrgico da obesidade, os tipos de procedimentos atualmente realizados e seus resultados.

Palavras chaves: cirurgia bariátrica, adolescentes, obesidade

Caravatto, Pedro Paulo; Petry, Tarissa; Cohen, Ricardo. "Cirurgia bariátrica em adolescentes", in Anais do 2‥ Congresso Internacional Sabará de Especialidades Pediátricas

\section{INTRODUÇÃO}

O presente capítulo visa abordar o tratamento cirúrgico do adolescente obeso mórbido, tema que vem ganhando popularidade nos últimos anos, principalmente devido ao aumento da prevalência da obesidade nesta faixa etária.

Para análise do grau de obesidade na infância e na adolescência utiliza-se o gráfico índice de massa corpórea (IMC) por idade (figura 1). Define-se sobrepeso quando o IMC encontra-se acima do 
percentil 85, enquanto que obesidade e obesidade mórbida são definidas quando o IMC encontra-se acima do percentil 95 e 99, respectivamente. A utilização do gráfico faz-se necessária, uma vez que o IMC, embora uma boa ferramenta para avaliação antropométrica populacional, quando utilizado isoladamente, tem menor utilidade na avaliação da criança e do adolescente, pois as variações de peso e altura são esperadas nesta faixa etária.

\section{EPIDEMIOLOGIA}

No Brasil, dados do Instituto Brasileiro de Geografia e Estatística (IBGE) apontam para um crescimento dramático na prevalência da obesidade em crianças e adolescentes entre os anos de 1989 e 2009, sendo observados aumentos de até 500\% dependendo da faixa etária (figura 2).

Nos EUA, observa-se tendência semelhante. Em três décadas, a prevalência da obesidade em crianças de 6 a 11 anos aumentou de 7 para 18\%, enquanto que em adolescentes de 12 a 19 anos, aumentou de 5 para $18,4 \%$ no mesmo período. 1

Ao estratificar o grau de obesidade, os achados são mais alarmantes, uma vez que se observa crescimento mais acentuado entre crianças e adolescentes obesos mórbidos, que representam atualmente 4,7\% das crianças com idade entre 6 e 11 anos e 6,3\% dos adolescentes (12 a 19 anos). 2

\section{IMPACTO DA OBESIDADE}

Uma das principais preocupações é o impacto que a obesidade na adolescência exerce na vida adulta. Sabe-se que crianças e adolescentes obesos têm maior chance de serem obesos quando adultos. Além disso, frequentemente são vítimas de bullying e preconceito, aumentado o risco de depressão e levando à piora da qualidade de vida.

Além disso, a obesidade na infância acarreta diversas comorbidades (tabela 1) que tendem a agravar ao longo da vida adulta. Dentre elas, a síndrome da apnéia obstrutiva do sono acomete mais de 50\% dos adolescentes obesos mórbidos. Esteatose hepática, por sua vez, apresenta prevalência em torno de $80 \%$, enquanto que $20 \%$ dos adolescentes obesos mórbidos já apresentam esteatohepatite. A intolerância à glicose, associada a hiperinsulinemia, importante fator de risco cardiovascular entre adultos, é 3 a 5 vezes mais frequente em adolescentes obesos mórbidos quando comparada em adolescentes obesos não mórbidos.

O grau de obesidade do adolescente é diretamente proporcional ao número de fatores de risco para doenças cardiovasculares em adultos (hiperinsulinemia, elevação da pressão arterial sistólica ou diastólica, hipercolesterolemia com redução dos níveis de HDL e hipertrigliceridemia). 
Aproximadamente $11 \%$ dos adolescentes obesos mórbidos apresenta 4 ou mais fatores de risco, enquanto que $84 \%$ apresenta pelo menos um fator de risco. 3

\section{TRATAMENTO CLÍNICO}

O tratamento clínico da obesidade a base de dieta hipocalórica e estímulo à prática de atividade física apresenta resposta insatisfatória, semelhante à população adulta. $\mathrm{O}$ uso de medicamentos para auxiliar a redução de peso nesta faixa etária é controverso e não será abordado no presente capítulo.

A baixa eficácia deste tipo de tratamento pode ser comprovada em estudo longitudinal avaliando os efeitos do tratamento clínico não medicamentoso em adolescentes obesos mórbidos. Observou-se aumento médio do IMC em 6\% após 7 anos de acompanhamento periódico visando estimular a prática de atividade física e consumo de alimentos hipocalóricos com baixo índice glicêmico. 4

\section{RACIONAL DO TRATAMENTO CIRÚRGICO}

Desta forma, frente à ineficácia do tratamento clínico, a cirurgia bariátrica tornou-se cada vez mais popular em adolescentes, principalmente após os bons resultados quanto à perda de peso e à resolução das comorbidades observadas na população adulta. Além disso, os baixos índices de morbidade e mortalidade, principalmente após o aprimoramento da técnica cirúrgica, contribuíram para o aumento do número de procedimentos realizados.

Dados da Sociedade Americana de Cirurgia Bariátrica e Metabólica mostram que nos últimos anos o número de procedimentos bariátricos em adolescentes quintuplicou, sendo realizados mais de 4000 procedimentos anualmente em adolescentes.

Os resultados observados em cirurgia bariátrica nos adultos mostram que quanto maior a duração da obesidade e a gravidade das doenças associadas, menor é a probabilidade de resolução dos mesmos após a cirurgia. Desta forma, questiona-se atualmente qual o melhor momento para a cirurgia bariátrica no adolescente.

De acordo com a portaria 424 de 19 de março de 2013 do Ministério da Saúde, a cirurgia bariátrica poderá ser realizada em adolescentes com idade entre 16 e 18 anos somente após análise cuidadosa do risco e benefício deste tipo de tratamento, desde que a consolidação das epífises de crescimento tenham ocorrido. Por outro lado, acredita-se que a cirurgia não deveria ser retardada, especialmente em adolescentes obesos mórbidos com comorbidades graves, uma vez que ao ser realizada mais precocemente, diminui o risco de complicações e aumenta a probabilidade de bons resultados, diminuindo o risco de recidiva e a probabilidade de reoperações por perda de peso insuficiente. 5 


\section{CRITÉRIOS DE INDICAÇÃO DE CIRURGIA BARIÁTRICA EM ADOLESCENTES}

Atualmente, os critérios de indicação para cirurgia bariátrica em adolescentes levam em conta o IMC, à semelhança dos adultos. Assim sendo, a cirurgia é indicada para os pacientes com IMC $>40 \mathrm{~kg} / \mathrm{m} 2$ ou IMC $>35 \mathrm{~kg} / \mathrm{m} 2$ quando associado a pelo menos uma comorbidade de difícil controle clínico, tais como diabetes tipo 2, hipertensão arterial, apnéia obstrutiva do sono, dentre outras.

Nesta população, é importante destacar a necessidade de atingir a maturidade puberal (estágio IV ou V de Tanner) e/ou desenvolvimento físico avaliado pela idade óssea (aproximadamente 95\% da altura do indivíduo adulto com idade óssea semelhante). Avaliando esses critérios e considerando a idade do adolescente, observa-se que a maioria dos adolescentes do sexo masculino acima dos 15 anos e do sexo feminino acima dos 13 anos, já atingiram esses critérios de desenvolvimento físico/puberal, o que autorizaria a realização do tratamento cirúrgico em uma população mais jovem do que a atualmente proposta pela legislação. Tal observação só poderá ser validada após a divulgação de maior número de dados de estudos randomizados e controlados, atestando a segurança e eficácia da cirurgia bariátrica em uma população mais jovem.

A capacidade de compreender as mudanças de comportamento e hábitos de vida, visando melhor resultado a longo prazo é fundamental. Assim sendo, o adolescente deve estar apto a tomar decisões, compreendendo os riscos e benefícios da cirurgia. Para tanto, deverá contar com o acompanhamento de uma equipe multidisciplinar. Além disso, o apoio familiar nesta fase é igualmente importante. Nos casos de doenças psiquiátricas, estas deverão ser tratadas e acompanhadas por psiquiatra, podendo a cirurgia ser indicada apenas quando estiverem controladas.

O leitor mais atento poderá questionar a esta altura o porquê de um tratamento invasivo, senão drástico, (muitas vezes definitivo) em uma população tão jovem, alguns com 13 anos conforme proposto anteriormente.

De pronto, questiona-se os termos invasivo e drástico como adjetivos da cirurgia bariátrica. De forma alguma os resultados atuais da cirurgia preconizam o uso destes termos. Artigo de revisão publicado pela Sociedade Americana de Cirurgia Bariátrica e Metabólica em conjunto com outras sociedades de diversas especialidades, observou que a morbidade da cirurgia bariátrica em adolescentes foi de 2,9\%, enquanto que não ocorreram óbitos nesta população. Estes resultados são muito inferiores aos observados em outros tipos de cirurgias pediátricas, como por exemplo cirurgias cardíacas, onde a mortalidade é de 5,4\%. 6 


\section{TIPOS DE CIRURGIA BARIÁTRICA E RESULTADOS}

Classicamente, a cirurgia bariátrica é dividida em procedimentos restritivos, mistos e disabsortivos. Dentre os procedimentos restritivos, a banda gástrica tem sido abandonada, principalmente pelos maus resultados a médio e longo prazo observados na população adulta, e praticamente não é realizada em adolescentes. 7

Os procedimentos disabsortivos (derivações bilio-pancreáticas com ou sem exclusão duodenal), uma vez que apresentam um índice elevado de complicações nutricionais a longo prazo, são contraindicados em adolescentes.

Desta forma, as principais opções de cirurgia bariátrica em adolescentes são a gastrectomia vertical (GV) e a gastroplastia em Y Roux (GYR). (Figura 3)

Para efeitos comparativos, a GV, embora apresente resultados comparáveis aos observados após GYR nos primeiros 2 anos, não possui resultados a longo prazo. Por ser um procedimento relativamente recente, carece de seguimento por tempo mais longo. Além disso, é um procedimento definitivo, uma vez que, nesta cirurgia, parte do estômago (fundo e parte do corpo e antro) é ressecada, de forma a confeccionar um tubo longitudinal a fim de conferir restrição ao procedimento. Sabe-se que existem mecanismos metabólicos inerentes a este procedimento, embora seus efeitos a longo prazo sejam questionáveis. De qualquer forma, quando comparada a procedimentos puramente restritivos, como a banda gástrica, os resultados da GV são superiores, embora sejam inferiores aos observados na GYR. (Tabela 2)

A GYR apresenta bons resultados com relação à perda de peso e resolução das comorbidades, implicando em redução da mortalidade, principalmente quando avaliadas complicações referentes ao diabetes tipo 2. Neste caso, observa-se redução de $92 \%$ na mortalidade relacionada ao diabetes na população adulta submetida a GYR. 9

Resultados semelhantes na população adolescente foram observados após GYR conforme relatado por Olber e cols. Em seguimento de dois anos em 100\% dos pacientes, os autores reportaram redução de 32\% do IMC (76\% do excesso), resultado semelhante ao observado na população adulta (redução de 31\% do IMC) e superior ao tratamento clínico (dieta hipocalórica e prática de atividade física), em que se observou ganho 3\% do peso em adolescentes. Além disso, houve melhora significativa de conhecidos fatores de risco cardiovascular, tais como níveis de insulina e proteína c-reativa. 10

\section{CONCLUSÕES}


A obesidade infantil vem aumentanto progressivamente, atingindo uma população cada vez mais jovem. Consequentemente, comorbidades típicas de adultos vem sendo observadas em adolescentes com uma frequencia cada vez maior, o que pode, a longo prazo, trazer graves complicações.

É neste cenário que a cirurgia bariátrica consolida-se como alternativa eficaz quando medidas clínicas falham. Soma-se ao fato de ser extremamente segura, com baixo índice complicações e elevado indice de sucesso, semelhante aos observados em adultos, podendo adicionar anos de vida com qualidade. 


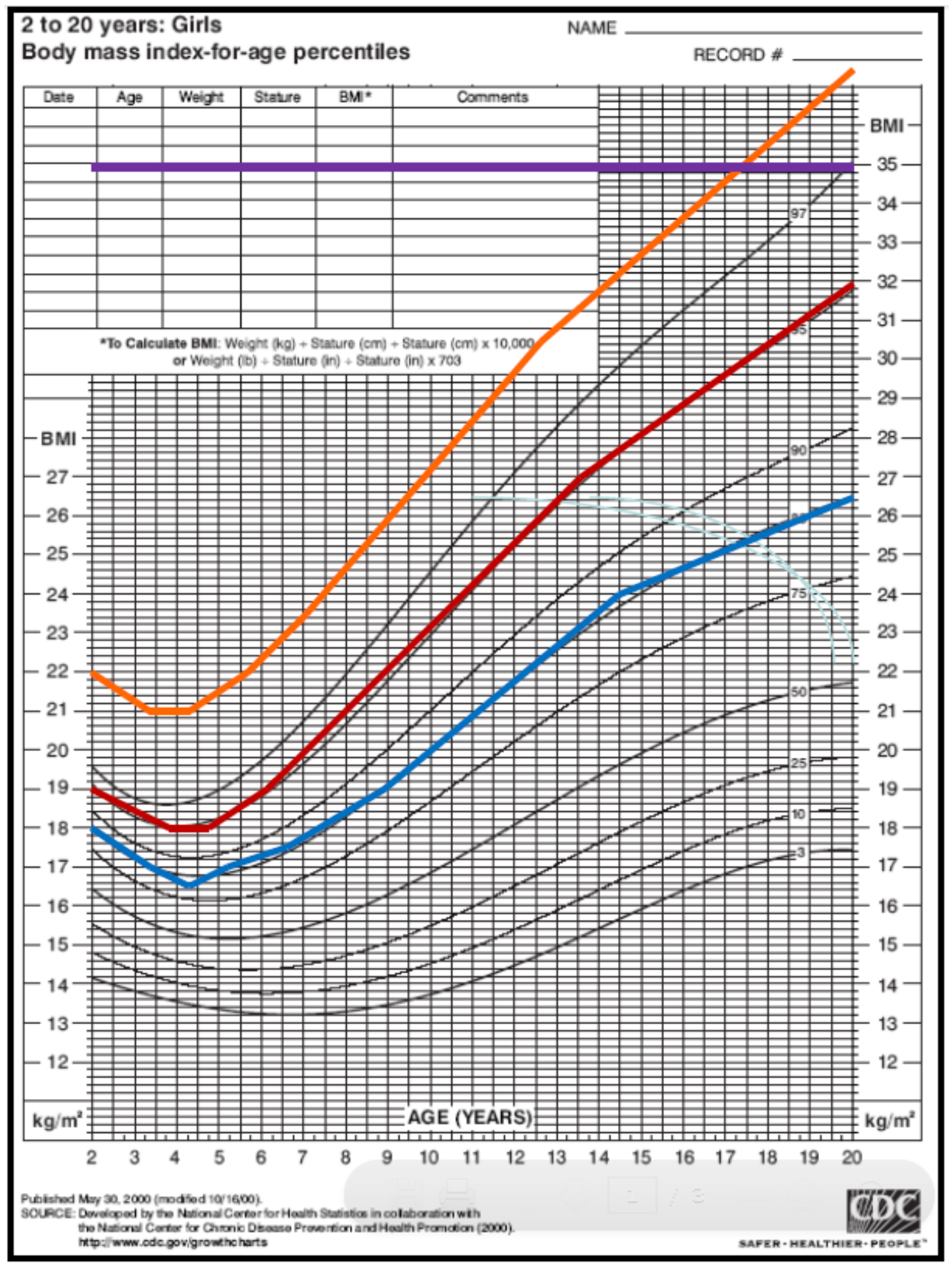

Figura 1 - IMC x idade para o sexo feminino (idade 2 a 20 anos). Os percentis para sobrepeso, obesidade e obesidade mórbida (85, 95 e 99, respectivamente) encontram-se destacados. 

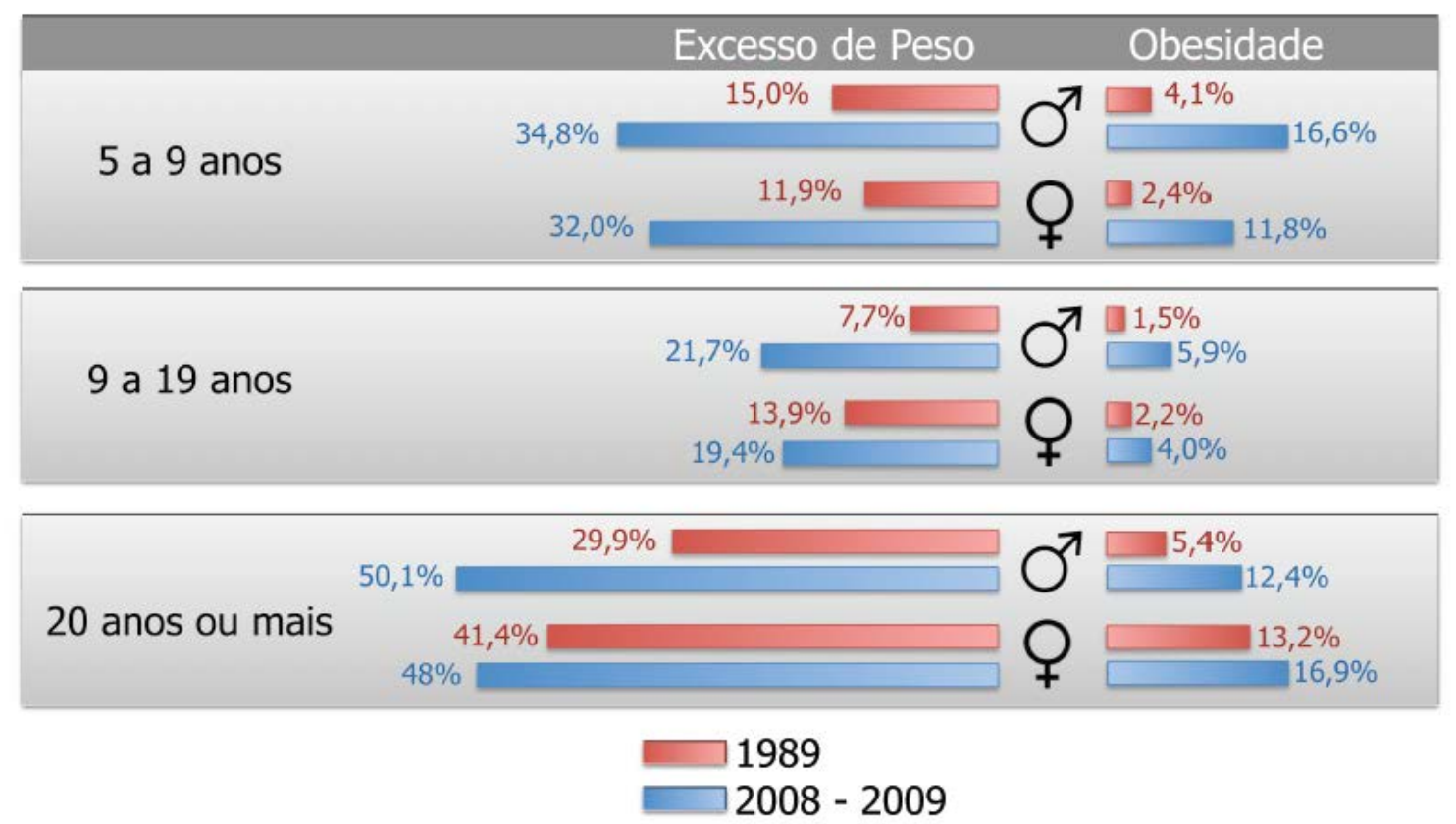

Figura 2 - Análise da prevalência da obesidade em crianças e adolescentes no Brasil entre os anos de 1989 e 2009 (Fonte: IBGE) 
Tabela 1 - Co-morbidades associadas à obesidade em adolescentes

\begin{tabular}{|l|l|}
\hline Pulmonar & Asma \\
\hline & Apnéia obstrutiva do sono \\
\hline Renal & Intolerância aos exercícios \\
\hline Gastrointestinal & Glomeruloesclerose \\
\hline & Proteinúria \\
\hline & Paniculite \\
\hline Osteoarticular & Esteatohepatite \\
\hline & Fibrose hepatica \\
\hline & Colelitíase \\
\hline Endocrinológico & Maior risco de cancer \\
\hline & Fraturas de antebraço \\
\hline Neurológico & Doença de Blount \\
\hline & Pé chato \\
\hline Cardiovascular & Maior risco de degeneração articular \\
\hline & Pseudotumor cerebri \\
\hline & Maior risco de acidente vascular cerebral \\
\hline & Hipertensão arterial \\
\hline & Dislipidemia \\
\hline & Hipertrofia do ventrículo esquerdo \\
\hline & Inflamação crônica \\
\hline & Disfunção do endotélio \\
\hline & Maior risco de doença coronariana \\
\hline & Diabetes tipo 2 \\
\hline
\end{tabular}




\begin{tabular}{|l|l|}
\hline & Síndrome ovário policístico (meninas) \\
\hline & Hipogonadismo (meninos) \\
\hline
\end{tabular}




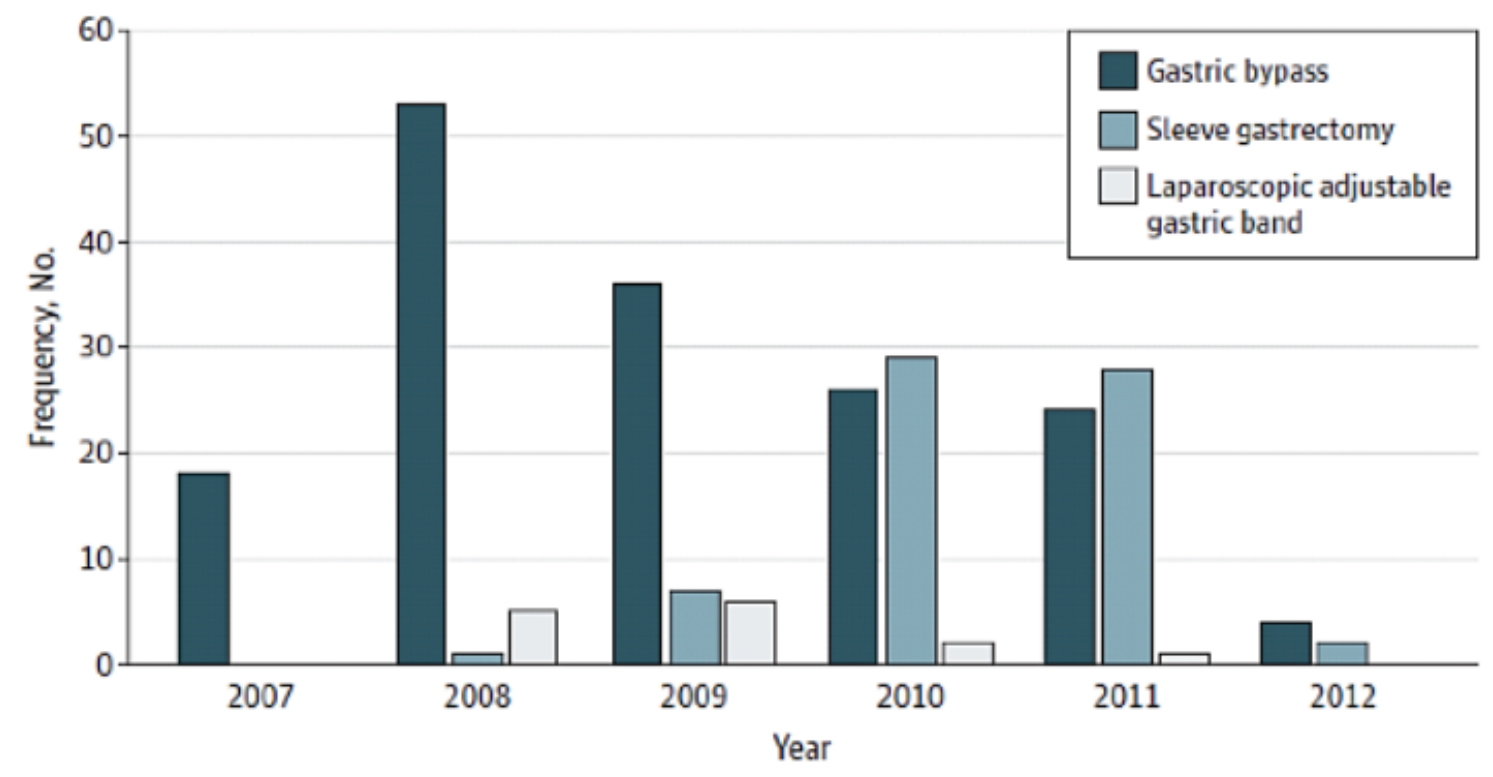

Figura 3. Tipos de cirurgia bariátrica em adolescentes. Extraído de Hinge et al. JAMA Pediatrics online, 2003. 
Tabela 2 - Resultados da cirurgia bariátrica em adolescentes. Adaptado de Black et al. Obesity $2013^{8}$

\begin{tabular}{|l|l|l|l|}
\hline $\begin{array}{l}\text { Tipo de } \\
\text { procedimento }\end{array}$ & Tipo de estudo & Número de pacientes & Redução do IMC \\
\hline $\begin{array}{l}\text { Banda gástrica } \\
\text { ajustável }\end{array}$ & Meta-análise & 271 & $10,5 \mathrm{~kg} / \mathrm{m}^{2}$ \\
\hline Gastrectomia vertical & Meta-análise & 90 & $14,7 \mathrm{~kg} / \mathrm{m}^{2}$ \\
\hline $\begin{array}{l}\text { Gastroplastia Y } \\
\text { Roux }\end{array}$ & Meta-análise & 256 & $17,2 \mathrm{~kg} / \mathrm{m}^{2}$ \\
\hline
\end{tabular}

\section{ABSTRACT}

Pediatric obesity has become a serious problem in public health. Obesity related diseases commonly observed in adults, such as diabetes, hypertension and dyslipidemia, have become increasingly prevalent in teenagers and will have a significantly decrease in life expectancy.

Such problem prompts immediate action, such as surgical treatment for obesity at an earlier stage in life in order to avoid adverse outcomes in the long-term. Bariatric surgery in adolescents has its particular risks and benefits that must be accounted for, especially when dealing with unique psychological and emotional needs of the adolescent.

Initial outcomes of bariatric surgery in adolescents are promising and available data show they are comparable to those seen in adults. However, long-term data are still needed.

In this chapter, the authors discuss the epidemiology and burden of childhood obesity, indications for bariatric surgery in adolescents, types of procedures that are currently being performed and reported outcomes.

Keywords: bariatric surgery, adolescents, obesity 


\section{REFERÊNCIAS}

1. Ogden CL, Freedman DS. Secular trends in pediatric BMI. Am J Clin Nutr 2012; 95(5): 999-1000.

2. Beydoun MA, Wang Y. Socio-demographic disparities in distribution shifts over time in various adiposity measures among American children and adolescents: What changes in prevalence rates could not reveal. Int J Pediatr Obes 2011; 6(1): 21-35.

3. Freedman DS, Mei Z, Srinivasan SR et al. Cardiovascular risk factors and excess adiposity among overweight children and adolescents: the Bogalusa Heart Study. J Pediatr 2007; 150(1): 12-7.

4. Hipsky J, Kirk S. HealthWorks! Weight management program for children and adolescents. J Am Diet Assoc 2002; 102(3): S64-7.

5. Garcia VF, DeMaria EJ. Adolescent bariatric surgery: treatment delayed, treatment denied, a crisis invited. Obes Surg 2006; 16(1): 1-4.

6. Mechanick JI, Youdim A, Jones DB et al. Clinical practice guidelines for the perioperative nutritional, metabolic, and nonsurgical support of the bariatric patient - 2013 update: cosponsored by American Association of Clinical Endocrinologists, the Obesity Society, and American Society for Metabolic and Bariatric Surgery. Surg Obes Relat Dis 2013; 9(2): 159-91.

7. Inge $\mathrm{TH}$, Zeller $\mathrm{MH}$, Jenkins $\mathrm{TM}$ et al. Perioperative outcomes of adolescents undergoing bariatric surgery: the Teen-Longitudinal Assessment of Bariatric Surgery (Teen-LABS) study. JAMA Pediatr 2014; 168(1): 47-53.

8. Black JA, White $B$, Viner RM et al. Bariatric surgery for obese children and adolescents: a systematic review and meta-analysis. Obes Rev 2013; 14(8): 634-44.

9. Adams TD, Gress RE, Smith SC et al. Long-term mortality after gastric bypass surgery. N Engl J Med 2007; 357(8): 753-61.

10. Olbers T, Gronowitz E, Werling $M$ et al. Two-year outcome of laparoscopic Roux-en-Y gastric bypass in adolescents with severe obesity: results from a Swedish Nationwide Study (AMOS). Int J Obes (Lond) 2012; 36(11): 1388-95. 